\title{
Cellular Uptake of Cationic Polymer-DNA Complexes Via Caveolae Plays a Pivotal Role in Gene Transfection in COS-7 Cells
}

\author{
M. A. E. M. van der Aa, ${ }^{1}$ U. S. Huth, ${ }^{2}$ S. Y. Häfele, ${ }^{2}$ R. Schubert, ${ }^{2}$ R. S. Oosting, ${ }^{3}$ E. Mastrobattista, ${ }^{1,5}$ \\ W. E. Hennink, ${ }^{1}$ R. Peschka-Süss, ${ }^{2}$ G. A. Koning, ${ }^{4}$ and D. J. A. Crommelin ${ }^{1}$
}

Received January 5, 2007; accepted February 27, 2007; published online March 24, 2007

Purpose. Knowledge about the uptake mechanism and subsequent intracellular routing of non-viral gene delivery systems is important for the development of more efficient carriers. In this study we compared two established cationic polymers pDMAEMA and PEI with regard to their transfection efficiency and mechanism of cellular uptake.

Materials and Methods. The effects of several inhibitors of particular cellular uptake routes on the uptake of polyplexes and subsequent gene expression in COS-7 cells were investigated using FACS and transfection. Moreover, cellular localization of fluorescently labeled polyplexes was assessed by spectral fluorescence microscopy.

Results. Both pDMAEMA- and PEI-complexed DNA showed colocalization with fluorescently-labeled transferrin and cholera toxin after internalization by COS-7 cells, which indicates uptake via the clathrinand caveolae-dependent pathways. Blocking either routes of uptake with specific inhibitors only resulted in a marginal decrease in polyplex uptake, which may suggest that uptake routes of polyplexes are interchangeable. Despite the marginal effect of inhibitors on polyplex internalization, blocking the caveolae-mediated uptake route resulted in an almost complete loss of polyplex-mediated gene expression, whereas gene expression was not negatively affected by blocking the clathrin-dependent route of uptake.

Conclusions. These results show the importance of caveolae-mediated uptake for successful gene expression and have implications for the rational design of non-viral gene delivery systems.

KEY WORDS: cationic polymers; caveolae; endocytosis; gene delivery.

\section{INTRODUCTION}

Polyplexes are non-viral gene delivery systems based on cationic polymers that are able to condense the DNA into small particles. Due to the net positive surface charge these polyplexes efficiently bind to the cell through electrostatic interactions with negatively charged membrane components. Although the molecular details of the mechanism by which the cationic polyplexes mediate DNA delivery are still poorly understood, current evidence suggests that the positively charged polyplexes are taken up by means of endocytosis (1-3).

\footnotetext{
${ }^{1}$ Department of Pharmaceutics, Utrecht Institute for Pharmaceutical Sciences (UIPS), Utrecht University, P.O. Box 80082, 3508 TB, Utrecht, The Netherlands.

${ }^{2}$ Department of Pharmaceutical Technology and Biopharmacy, Albert-Ludwigs University, Stefan-Meier-Str. 19, D-79104, Freiburg, Germany.

${ }^{3}$ Department of Psychopharmacology, Utrecht Institute for Pharmaceutical Sciences (UIPS), Utrecht University, P.O. Box 80082, 3508 TB, Utrecht, The Netherlands.

${ }^{4}$ Laboratory of Experimental Surgical Oncology, Department of Surgical Oncology, Erasmus MC, Erasmus University, PO Box 1738, 3000 DR, Rotterdam, The Netherlands.

${ }^{5}$ To whom correspondence should be addressed. (e-mail:E.Mastrobattista @pharm.uu.nl)
}

Since different endocytic pathways exist, it is important to have a more detailed knowledge of the cellular uptake and routing mechanism of polyplexes since each pathway has its own characteristics, which should be taken into account when optimizing transfection efficiency of gene delivery systems (4). The best-characterized uptake mechanism is the clathrindependent pathway, which carries the polyplexes into early and late endosomes that ultimately fuse with lysosomes and the trans-golgi network (5). The non-clathrin dependent endocytosis pathways include caveolin-mediated endocytosis, macropinocytosis, clathrin- and caveolin-independent endocytosis and phagocytosis. Whereas the latter is restricted to specialized cells, the other pathways can occur in any cell type. Caveolae are cholesterol- and sphingolipid rich smooth invaginations of the plasma membrane that partition into raft fractions. Their occurrence is associated with caveolin-1 (6, 7). Caveolae are subdomains of glycolipid rafts and are internalized via a common, clathrin independent, dynamin dependent and cholesterol sensitive pathway (8). Caveolae and raft pathways mediate the internalization of certain viruses, such as Simian Virus 40, bacteria and sphingolipid binding toxins, like the cholera toxin (9-11). Recently, it has been shown that particles taken up by the caveolae-mediated pathway are delivered to caveosomes, which are pre-existing, stable, organelles with a neutral $\mathrm{pH}$ and multiple flask- 
shaped caveolar domains enriched in caveolin-1 (12). Macropinocytosis is a triggered process used by the cell to internalize large amounts of fluid and membrane. Macropinocytosis is characterized by the formation of large, irregular primary endocytic vesicles after closure of ruffling membrane domains. Macropinosomes are dynamic structures that frequently move inwards towards the center of the cell (13).

Intracellular trafficking of polyplexes has been widely studied using fluorescent labels. Most studies involve the simultaneous use of several fluorophores to determine the intracellular location. A method to distinguish between these different dyes involves a spectral imaging technique. This enables the simultaneous measurement of the fluorescence spectrum for each pixel of the microscope image without changing the filter set. Subsequent analysis of the measured data results in the exact localization of each dye in the image (14).

In the present study the uptake mechanism, intracellular fate and gene expression of polyplexes based on two different cationic polymers, polyethyleneimine (PEI) and poly(2(dimethylamino)ethyl methacrylate (pDMAEMA) were investigated. Both polymers are able to condense DNA and facilitate gene delivery $(15,16)$. However, detailed knowledge is lacking regarding the exact mechanism of uptake, subsequent intracellular routing and ultimate gene expression. Intracellular localization of polyplexes has been studied using fluorescently labeled polyplexes $(17,18)$ and both pDMAEMA- and PEI-based polyplexes have been found in lysosomal vesicles $(17,19)$. An escape mechanism from these lysosomes was postulated for PEI based on its buffering capacity. The $\mathrm{pH}$ drop in PEI-loaded endosomes leads to osmotic swelling and bursting of the vesicle; the so-called proton sponge effect $(15,20,21)$. Recently, it was observed that more than one endocytotic pathway is involved in polyplex uptake. For example, Huth et al. showed, by the use of specific inhibitors of various endocytic pathways, that PEI polyplexes are taken up both by clathrin-dependent and caveolae-mediated endocytosis in HeLa cells (22) while Rejman et al. obtained similar results in HeLa and A549 cells (23). Additionally, Grosse et al. observed large PEI polyplexes in macropinocytosis, intermediate (100-200 nm) polyplexes in clathrin-coated pits and small particles in caveolae using electron microscopy to examine intracellular trafficking of complexes in human airway epithelial cells (24).

In this study, we have examined further the uptake mechanism of both PEI and pDMAEMA polyplexes in COS7 cells. By means of specific inhibitors of the different endocytic pathways we determined that both clathrin-dependent and independent routes are involved in the cellular uptake of these polyplexes in COS-7 cells. Furthermore, inhibition of either one of the uptake routes leads to increased uptake via the other route. However, only caveolae-dependent uptake results in efficient gene delivery and subsequent gene expression.

\section{MATERIALS AND METHODS}

All reagents were purchased from Sigma (Zwijndrecht, The Netherlands) unless stated otherwise. Linear poly(ethyleneimine) (PEI), ExGen 500, was purchased from MBI Fermentas (St Leon-Rot, Germany) and poly(2-(dimethylamino)ethyl methacrylate) (pDMAEMA) was synthesized as described before (16). Transferrin Alexa 488 and cholera toxin B Alexa 488 were obtained from molecular probes (Leiden, The Netherlands). PLuc was an expression plasmid encoding the firefly luciferase under the control of the human cytomegalovirus promoter (Plasmid factory, Bielefeld, Germany). The plasmid was labeled with the label IT CY5 nucleic acid labeling Kit (Mirus) according to the manufacturer's instructions. The rhodamine-B labeled plasmid (pGeneGrip Rhodamine/EGFP) was obtained from Gene Therapy Systems inc. (San Diego, USA).

\section{Cell Culture}

COS-7 African green monkey cells were grown in DMEM (Gibco BRL, Breda, The Netherlands) supplemented with antibiotics/antimycotics, 5\% heat-inactivated fetal bovine serum (Integro, Zaandam, The Netherlands) and $25 \mathrm{mM}$ HEPES. Cells were maintained at $37^{\circ} \mathrm{C}$ in a $5 \%$ $\mathrm{CO}_{2}$ humidified air atmosphere.

\section{Uptake Studies}

Two days before the uptake experiment 50,000 COS-7 cells were seeded per well in a 24 -well tissue culture plate. Immediately prior to incubation, the culture medium was replaced with $400 \mu \mathrm{l}$ DMEM medium, containing 10\% FCS. Polyplexes were prepared in hepes buffered saline (HBS) to obtain a final concentration of $1 \mu \mathrm{g}$ DNA/well. Polyplexes were prepared in a polymer/DNA N/P ratio of $5 / 1$ for pDMAEMA and 6/1 for PEI. pDMAEMA polyplexes were incubated for $30 \mathrm{~min}$ at room temperature and PEI polyplexes for $10 \mathrm{~min}$, as described by the manufacturer. For the timelapse experiment COS-7 cells were incubated for $60 \mathrm{~min}$ at $4^{\circ} \mathrm{C}$ with pDMAEMA- or PEI-based polyplexes containing plasmid DNA, which was covalently labeled with CY5 using a Mirus label it Kit followed by incubation at $37^{\circ} \mathrm{C}$ for $10,30,60$, 90, 120 or $180 \mathrm{~min}$. For inhibition experiments the cells were first incubated with one of the following inhibitors chlorpromazine $(56 \mu \mathrm{M})$, LY29004 $(50 \mu \mathrm{M})$, wortmannin $(50 \mathrm{nM})$, genistein $(200 \mu \mathrm{M})$ or nocodazole $(10 \mu \mathrm{M})$ for $60 \mathrm{~min}$ or methyl- $\beta$-cyclodextrin $(164 \mu \mathrm{M})$ for $15 \mathrm{~min}$ in completed medium prior to addition of polyplexes to the cells. pDMAEMA- or PEI-based polyplexes containing CY5labeled plasmid DNA were added and the cells were incubated at $37^{\circ} \mathrm{C}$ for another $60 \mathrm{~min}$. Subsequently, the cells were incubated with $200 \mu \mathrm{l}$ PBS, containing $100 \mathrm{ug} / \mathrm{ml}$ poly(laspartic acid) (pAspA) and $\pm 100 \mathrm{U} / \mathrm{ml}$ deoxyribonuclease I (DNAse) for $30 \mathrm{~min}$ at $4^{\circ} \mathrm{C}$. Finally, the cells were washed with PBS and incubated with trypsin/EDTA $(0.5 \mathrm{mg} / \mathrm{ml}$ trypsin, 0.2 $\mathrm{mg} / \mathrm{ml}$ EDTA) for $5 \mathrm{~min}$ at $4^{\circ} \mathrm{C}$ to detach the cells. The cells were harvested by centrifugation and the cell pellet was washed with and then resuspended in ice-cold PBS, containing $1 \%$ bovine serum albumin (BSA). The mean fluorescence intensity of 10,000 individual cells was measured with a FACS Calibur and analyzed using cell quest software (BD Biosciences).

\section{Spectral Bio-imaging}

COS-7 cells were seeded onto $12-\mathrm{mm}$ coverslips in 24-well plates 2 days before use. Polyplexes were prepared as described before, but using pGeneGrip Rhodamine (Gene Therapy 
Systems inc.) as plasmid DNA. The cells were washed twice with $1 \mathrm{ml}$ PBS and then incubated in $300 \mu \mathrm{l}$ of medium, containing the polyplexes and $5 \mu \mathrm{g} / \mathrm{ml}$ transferrin Alexa 488 or $1 \mu \mathrm{g} / \mathrm{ml}$ cholera toxin B Alexa 488, for $60 \mathrm{~min}$. Subsequently, the cells were fixed with $4 \%$ paraformaldehyde $(500 \mu \mathrm{l}, 10 \mathrm{~min}, \mathrm{RT})$ and the coverslips were mounted on glass slides with $3 \mu \mathrm{l}$ MobiGlow (MoBiTec, Goettingen, Germany), an antifading substance to reduce photobleaching effects.

Spectral imaging was performed with a SpectraCube SD$200 \mathrm{H}$ system (Applied Spectral Imaging, Migdal HaEmek, Israel), comprising of an inverted fluorescence microscope (Axiovert S 100, Zeiss) equipped with a high-pressure mercury lamp (HBO 100) for excitation and a triple bandpass filter set as described previously (14). All images were taken with a $100 \times / 1.3$ oil-immersion objective lens (Plan Neofluar, Zeiss) and captured using Spectral Imaging 2.5 software (Applied Spectral Imaging), the acquisition time of a desired image varied from 30 to $90 \mathrm{~s}$, depending on the brightness of fluorescence and the image size. First, cells were incubated with only one dye to get single-colored images. For further analysis, images were transferred to the SpectraView 1.6 software (Applied Spectral Imaging).

\section{Luciferase Transfection Study}

Twenty four hours before transfection 10,000 COS-7 cells were seeded per well into 96-well tissue culture plates, in order to reach a $60-70 \%$ confluence during transfection. Immediately prior to transfection the culture medium was refreshed with $100 \mu$ DMEM medium, containing 10\% FCS. Polyplexes were prepared as described above. For the timelapse experiment the cells were incubated with pDMAEMA- or PEI-complexed luciferase plasmid for $60 \mathrm{~min}$ at $4^{\circ} \mathrm{C}$. Subsequently, the cells were maintained at $37^{\circ} \mathrm{C}$ for $30 \mathrm{~min}, 1,1.5,2,3$, 4,8 or $24 \mathrm{~h}$, before being washed in ice cold PBS and harvested in $100 \mu \mathrm{l} 1 \mathrm{x}$ reporter lysis buffer (Promega). Of the cell suspension $20 \mu \mathrm{l}$ was diluted in $100 \mu \mathrm{l}$ luciferase reaction buffer (Promega) and the luminescence measured after $10 \mathrm{~s}$ using a luminometer (Berthold). Results were expressed as relative light units per mg of cell protein as determined by BCA protein assay (Pierce).

COS-7 cells were pretreated with chlorpromazine $(56 \mu \mathrm{M})$, LY29004 $(50 \mu \mathrm{M})$, wortmannin $(50 \mathrm{nM})$, nocodazole $(10 \mu \mathrm{M})$ or genistein $(200 \mu \mathrm{M})$ for $60 \mathrm{~min}$ or with methyl- $\beta$-cyclodextrin $(164 \mu \mathrm{M})$ for $15 \mathrm{~min}$ prior to addition of polyplexes to the cells. During transfection the cells were incubated in the presence of the inhibitors. The cells were incubated at $37^{\circ} \mathrm{C}$ with the polyplexes for $1 \mathrm{~h}$ in the presence of the inhibitors. Subsequently, the medium was refreshed with completed medium. The cells incubated with the polyplexes were maintained $24 \mathrm{~h}$ after transfection, washed in ice cold PBS and harvested in $100 \mu \mathrm{l} 1 \mathrm{x}$ reporter lysis buffer (Promega). Of the cell suspension $20 \mu \mathrm{l}$ was diluted in $100 \mu$ l luciferase reaction buffer (Promega) and the luminescence was measured after 10 s using a Lumat LB 9507 Berthold. Results were expressed as relative light units per mg of cell protein as determined by BCA protein assay (Pierce).

\section{Statistical Analysis}

The statistical analyses between different groups were determined with one-way ANOVA followed by a post hoc Dunnett $t$ test. A probability $p \leq 0.05$ was considered significant.

\section{RESULTS}

\section{Internalization and Transfection of Polyplexes in Time}

Polyplexes were prepared at a polymer/DNA (N/P) ratio of 5 for pDMAEMA and 6 for PEI polyplexes allowing for optimal transfection $(25,26)$. To test the uptake kinetics of the polyplexes, COS-7 cells were incubated with pDMAEMA and PEI complexed plasmid DNA. The DNA (pLuc) was covalently labeled with the fluorophore Cy5. First, COS-7 cells were incubated with the polyplexes at $4^{\circ} \mathrm{C}$ for $60 \mathrm{~min}$ to enable saturation of the binding of the polyplexes to the cell surface. Subsequently, the cells were washed, medium was added and the cells were incubated at $37^{\circ} \mathrm{C}$. After different incubation times the cells were treated with poly(1-aspartic acid) (pASPA) (27) and DNAse to remove the polyplexes still associated with the cell surface and internalized fluorescence was detected by FACS analysis. Effective removal of cell-bound polyplexes was validated with cells that remained at $4^{\circ} \mathrm{C}$ during the entire incubation period (results not shown). A rapid internalization of the PEI polyplexes was observed, while the internalization of the pDMAEMA polyplexes was much slower (Fig. 1a). Detectable internalization of pDMAEMA polyplexes was observed after $30 \mathrm{~min}$, whereas PEI polyplexes were internalized at an already detectable level within $10 \mathrm{~min}$. In fact, PEI polyplexes showed higher levels of internalization at all time points.

In a similar experimental setup luciferase gene expression was analyzed after incubation of the cells with PEI or pDMAEMA polyplexes for pre-selected periods of time. PEI-complexed DNA resulted in detectable levels of transfection already within $30 \mathrm{~min}$ of incubation, whereas pDMAEMA polyplex-mediated transfection was detected only after $90 \mathrm{~min}$ (Fig. 1b). For all time points luciferase expression was at least 5 to 10 -fold higher after incubation with PEI-complexed DNA than with pDMAEMA-complexed DNA.

\section{Co-localization of Polyplexes with Transferrin and Cholera Toxin}

To gain more insight into the intracellular fate of the polyplexes we used spectral bio-imaging to study co-localization of polyplexes with transferrin (Alexa 488-labeled), a marker for clathrin-mediated uptake $(28,29)$, or cholera toxin B (Alexa 488-labeled), which is thought to be internalized via a clathrinindependent route (30-32). COS-7 cells were incubated for $1 \mathrm{~h}$ with pDMAEMA or PEI polyplexes containing rhodamine labeled DNA. Spectral imaging was used to determine the intracellular localization of the different fluorescent labels. This method enables the simultaneous measurement of the fluorescence spectrum of each pixel of the microscopic image. Single color images were used to create the spectral library as was described before (14). pDMAEMA polyplexes were found in small vesicles throughout the cell (Fig. 2a and b), whereas PEI polyplexes were found in relatively large vesicles, about 5-10 times the size of the pDMAEMA containing vesicles (Fig. 3a and b). The same results were obtained when rhodamine-labeled pDMAEMA or PEI was used (data not shown). This difference in size of the vesicles containing the 


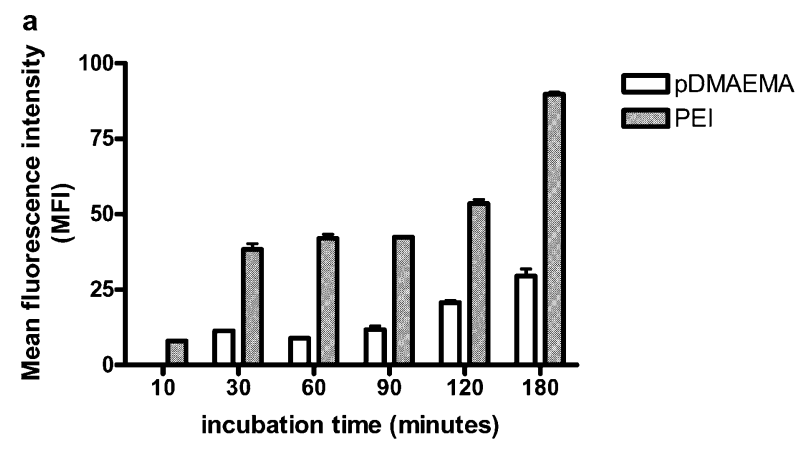

b

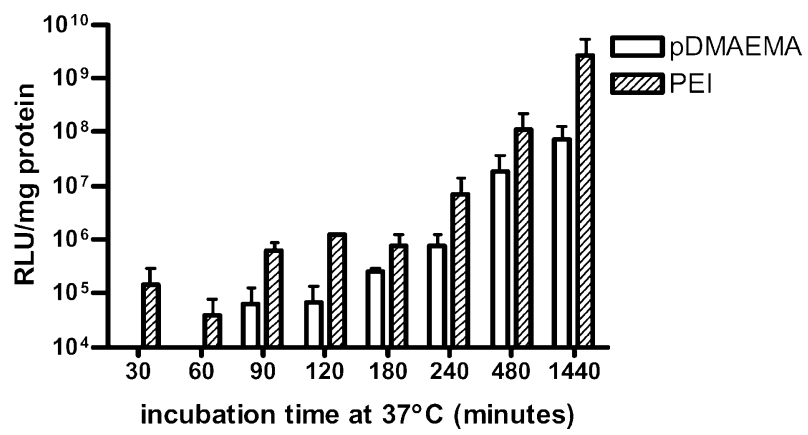

Fig. 1. Uptake (a) and transfection (b) of polyplexes in time. COS-7 cells were incubated with pDMAEMA- or PEI-based polyplexes for 60 minutes at $4^{\circ} \mathrm{C}$. Subsequently, the cells were incubated at $37^{\circ} \mathrm{C}$ and after different incubation times the internalized fluorescence (a) or the luciferase expression was determined (b). The open bars represent pDMAEMA- and the filled bars PEI-incubated cells. Results are means \pm SD from two different experiments.

polyplexes may be related to the size difference between the two polyplexes themselves. Under physiological conditions pDMAEMA polyplexes were approximately $200 \mathrm{~nm}$ and PEI polyplexes were approximately $1,000 \mathrm{~nm}$ with a broad size distribution as analyzed by photon correlation spectroscopy (Nicomp 380, Nicomp, Santa Barbara, USA). The intracellular location of pDMAEMA-based polyplexes was determined by investigating co-localization between the rhodamine-labeled DNA-containing polyplexes and transferrin (Alexa 488) or cholera toxin B (Alexa 488). Clear co-localisation of pDMAEMA polyplexes with transferrin and cholera toxin B was observed (Fig. 2). Co-localization between pDMAEMA and cholera toxin $\mathrm{B}$ was observed mainly in large vesicles (Fig. 2b). When cells were incubated with PEI-based polyplexes partial co-localization was observed between the polyplexes and transferrin Alexa 488 (Fig. 3a) as well as with cholera toxin B (Fig. 3b). This method, however, is not quantitative and provides no information on the amount of polyplexes, but it does show that PEI and pDMAEMA based polyplexes are taken up by clathrin-dependent and clathrinindependent endocytic routes.

\section{Quantitative Measurement of the Uptake of Polyplexes in the Presence of Endocytosis Inhibitors}

More quantitative data on the different uptake routes of PEI- and pDMAEMA-based polyplexes were obtained by monitoring internalization of particles in the presence of both general and specific inhibitors of endocytic routes using flow cytometry. Inhibitors were used at concentrations in which they have been shown to be active but not cytotoxic in COS-7 cells (33). The uptake of Cy5 labeled plasmid DNA, complexed by either pDMAEMA or PEI, was measured after dissociation and removal of polyplexes that were not internalized. Disruption of microtubuli by means of nocodazole decreased polyplex uptake to 70 and $50 \%$, as percentage of control (i.e. without pre-incubation with inhibitor), for pDMAEMA and PEI, respectively (Fig. 4a and b). It has been shown previously, that inhibition of macropinocytosis can be achieved by wortmannin and LY29004 (34, 35). In our experiments these substances did not reduce uptake of neither pDMAEMA nor PEI. These results demonstrate that macropinocytosis is not involved in the uptake of these polyplexes. Cholesterol depletion by methyl- $\beta$-cyclodextrin decreased the uptake to $50 \%$ for pDMAEMA and to $30 \%$ for PEI. Both caveolae- and clathrin-mediated endocytosis have been shown to depend on cholesterol (36-38). In order to distinguish between uptake via either one of these two routes we looked more closely at the effect of specific inhibition of these pathways. Inhibition of clathrin-mediated uptake by chlorpromazine did not significantly change the degree of internalization of pDMAEMA and PEI polyplexes. Higher concentrations of chlorpromazine were tested, but with similar results (data not shown). Inhibition of caveolaemediated uptake by genistein, an inhibitor of tyrosine kinases involved in caveolae-mediated endocytosis (39), did not significantly change the uptake of pDMAEMA-based polyplexes, but internalization of PEI polyplexes was reduced by almost $50 \%$. A combination of chlorpromazine and genistein resulted in a decrease in uptake of PEI polyplexes comparable to the effect of methyl- $\beta$-cyclodextrin. However, uptake of pDMAEMA polyplexes was not significantly decreased.

\section{Caveolar Routing is Important for Gene Expression}

To study the relation between the route of uptake and subsequent gene expression, COS-7 cells were incubated with polyplexes in the absence and presence of a variety of endocytosis inhibitors. Cells were pre-incubated with the inhibitor, before polyplexes were added and incubated for 1 $\mathrm{h}$ at $37^{\circ} \mathrm{C}$ in the presence of the same inhibitor. Afterwards, the polyplexes and inhibitors were removed and the cells were incubated at $37^{\circ} \mathrm{C}$ for another $24 \mathrm{~h}$ before luciferase activity was measured as readout for transfection efficiency (Fig. 5). Disruption of microtubuli by means of nocodazole decreased luciferase expression to $20 \%$ after transfection with pDMAEMA and to almost $0 \%$ when cells were incubated with PEI-based polyplexes. Indicating that both transport and to a lesser extent uptake requires microtubules as is shown in Fig. 4. Inhibition of macropinocytosis by wortmannin or LY29004, had no or even a positive effect on reporter gene expression, indicating that macropinocytosis did not contribute to gene delivery. Cholesterol depletion by methyl- $\beta$-cyclodextrin reduced luciferase expression levels of pDMAEMA-based polyplexes to $4 \%$ and of PEI polyplexes to $1 \%$. Inhibition of clathrin-mediated endocytosis by chlorpromazine had no significant effect on the transfection efficiency of both pDMAEMA and PEI polyplexes. Caveolae-mediated routing, on the other hand, 


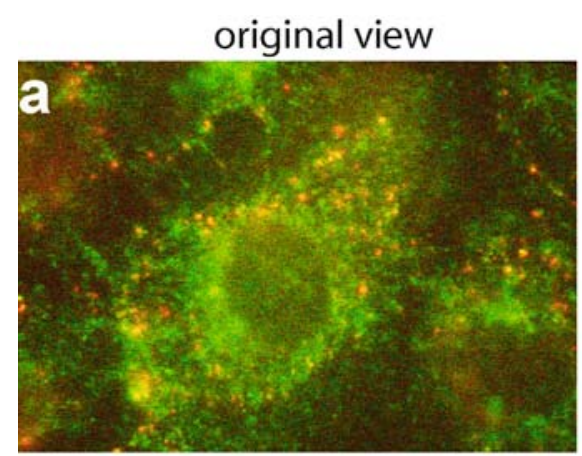

original view

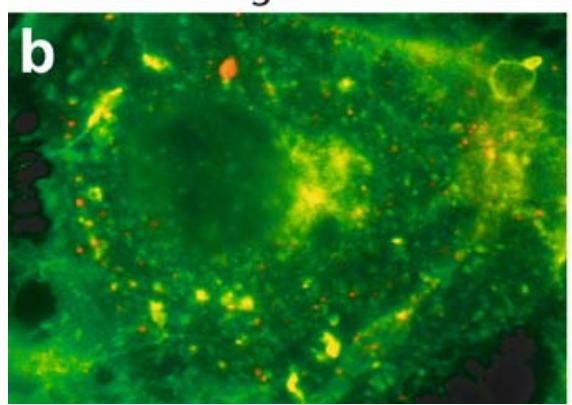

Transferrin

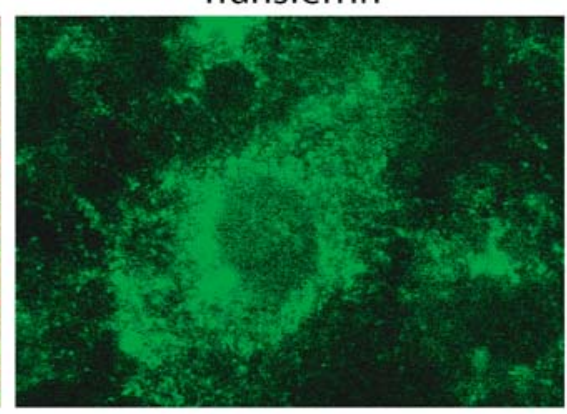

Cholera Toxin B

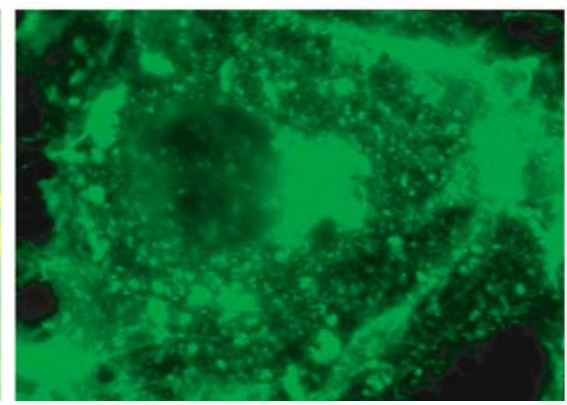

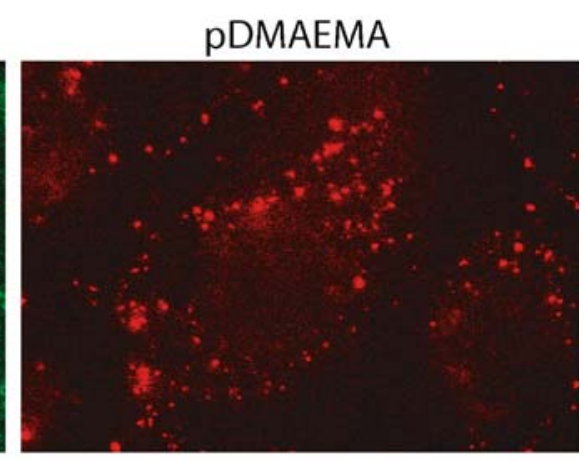

PDMAEMA

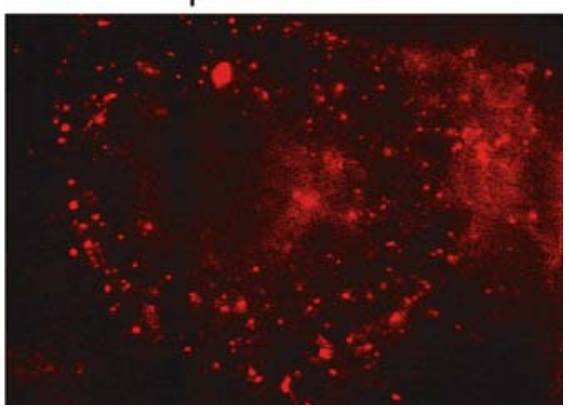

Fig. 2. Intracellular location of pDMAEMA-based polyplexes in COS-7 cells using spectral imaging. Polyplexes with rhodamine labeled DNA co-localized partly with transferrin alexa 488 (a) and partly with cholera toxin B Alexa 488 (b) after 1-h incubation. Representative pictures are displayed.

strongly contributed to gene expression. When this pathway was disturbed by genistein reporter gene expression was completely blocked for both PEI- and pDMAEMA-based polyplexes, demonstrating that caveolar routing is of crucial importance for gene expression even if uptake has not been reduced extensively. The observed reduction in gene expression is not caused by a direct toxic effect of the inhibitors as the presence of these inhibitors did not have a

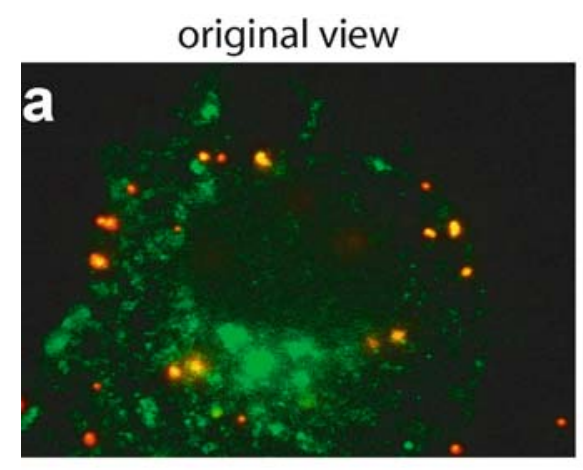

original view

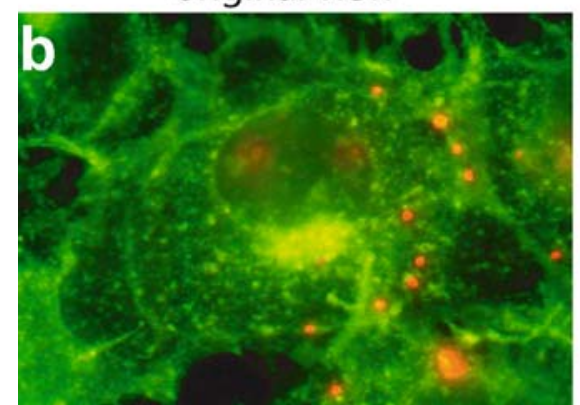

Transferrin

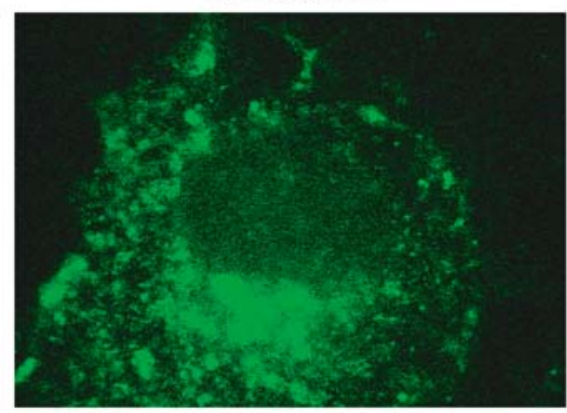

Cholera Toxin B

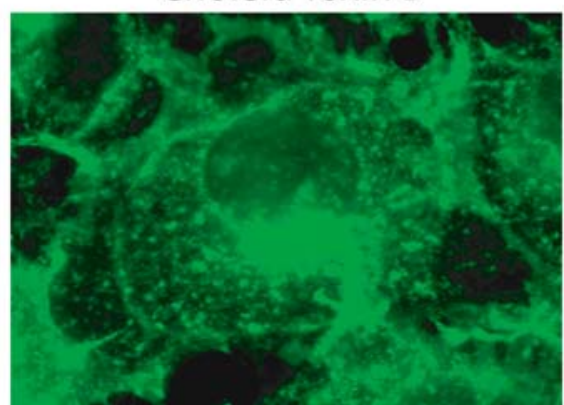

PEI

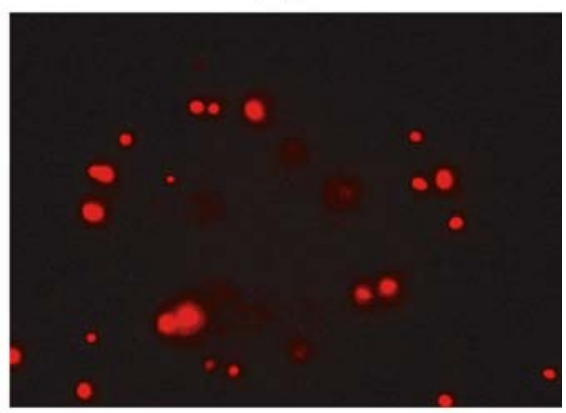

PEI

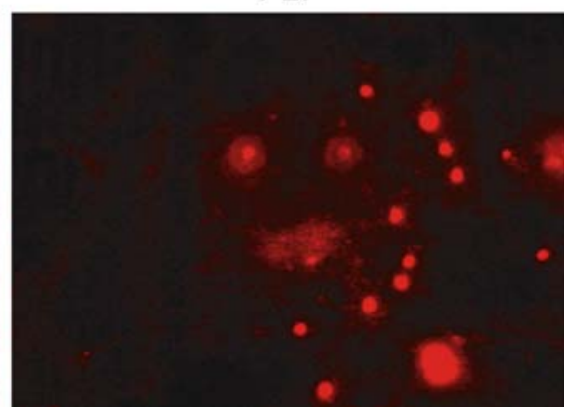

Fig. 3. Intracellular location of linear PEI-based polyplexes in COS-7 cells. With transferrin Alexa 488 clear co-localization is observed for rhodamine labeled DNA (a) the large PEI-based polyplexes also partly co-localize with cholera toxin B (b) after 1-h incubation. Representative pictures are displayed. 
negative effect on the luciferase expression in COS-7 cells that had been transiently transfected with the luciferase reporter plasmids prior to addition of the inhibitors (results not shown).
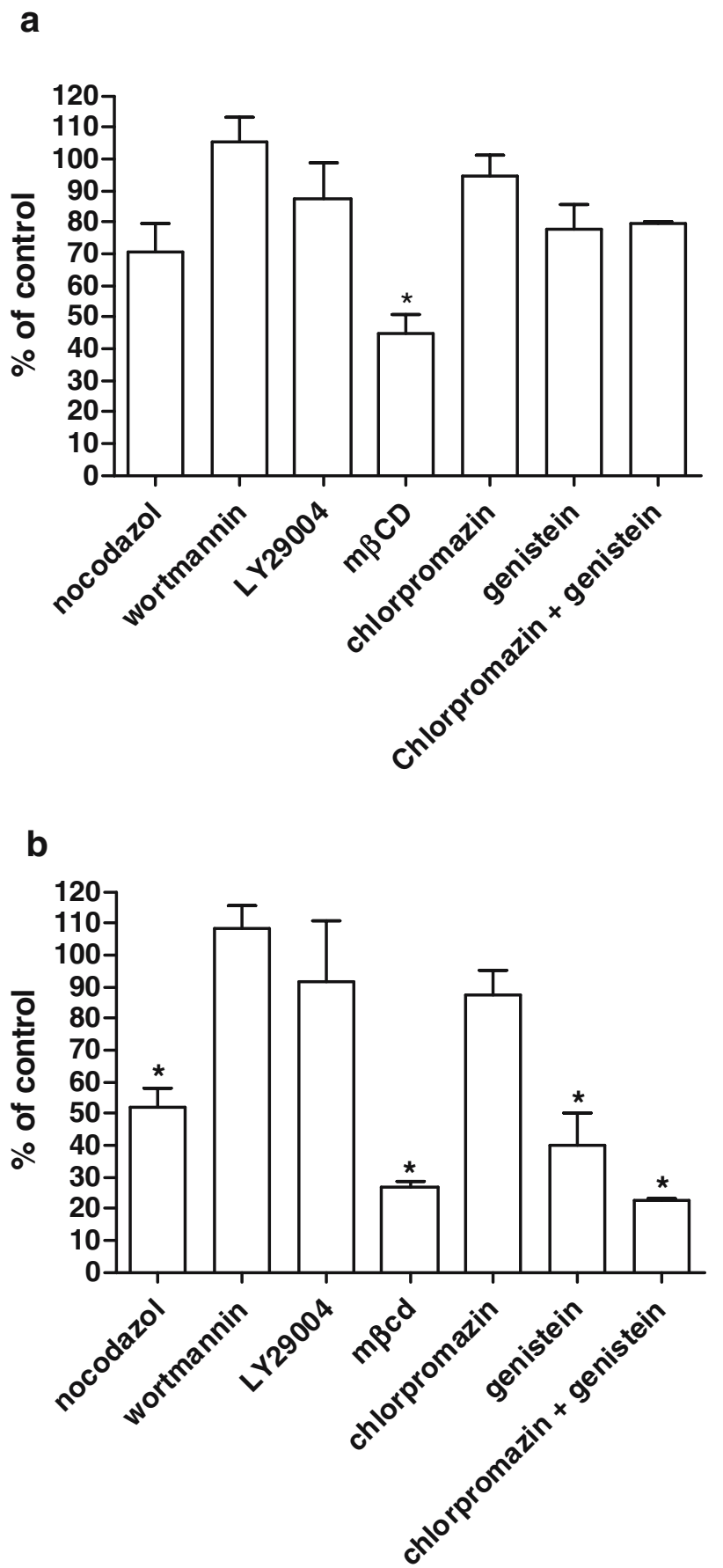

Fig. 4. Uptake of pDMAEMA (a) or PEI-based (b) polyplexes by COS-7 cells after microtubuli disruption (nocodazole), macropinocytosis inhibition (wortmannin and LY29004), cholesterol depletion $(\mathrm{m} \beta \mathrm{cd})$ or inhibition of clathrin- (chlorpromazine) or caveolaemediated (genistein) routing. Results, expressed as \% of control, are means $\pm \mathrm{SD}$ from three independent experiments. * Values significantly different from control $(p \leq 0.05)$.
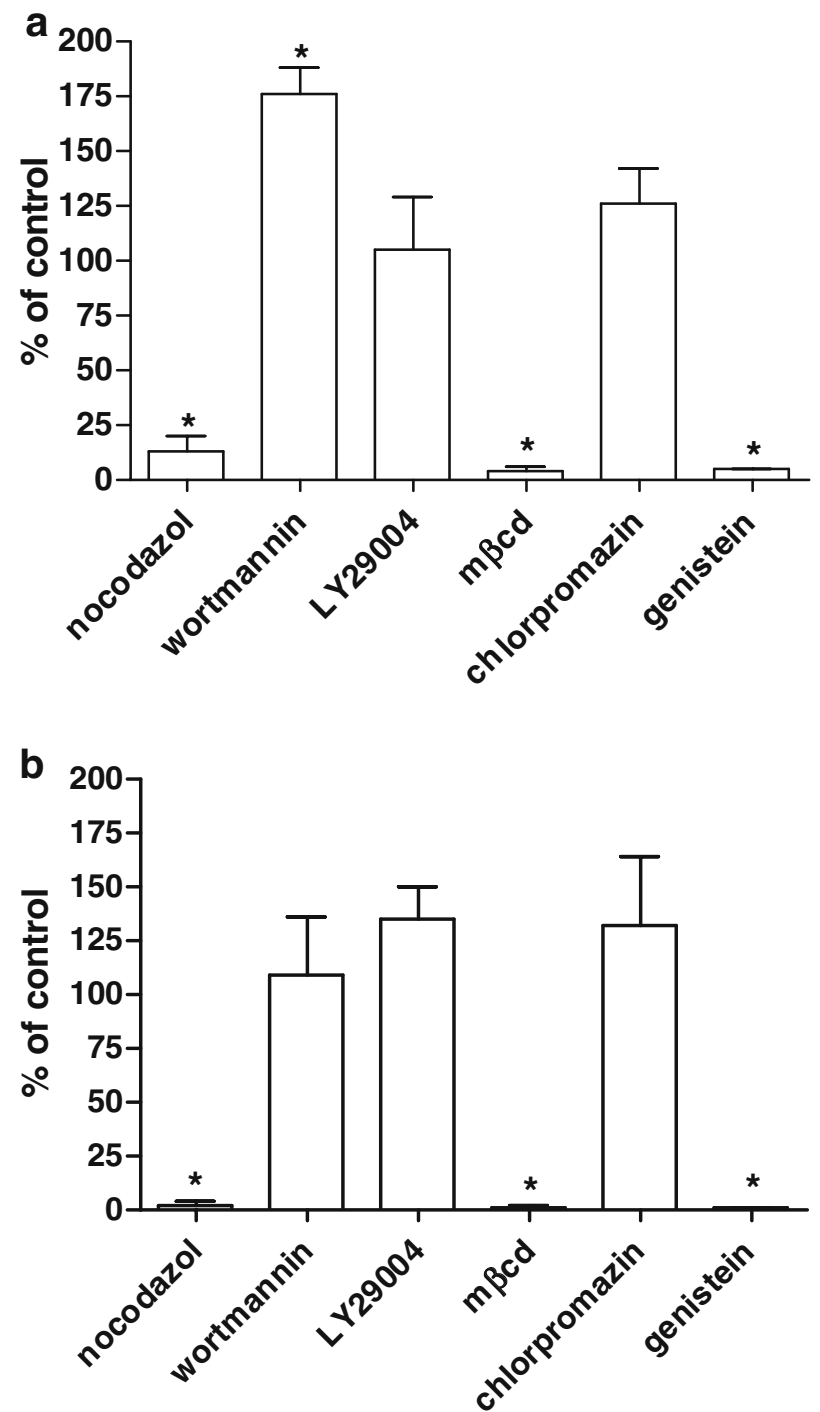

Fig. 5. Influence of endocytosis inhibitors on gene expression. Percentage of transfection of pDMAEMA- (a) or PEI-based (b) polyplexes after incubation with various inhibitors. Results are means \pm SD from at least four independent experiments. * Values significantly different from control $(p \leq 0.05)$.

\section{DISCUSSION}

Knowledge about the intracellular fate of non-viral gene delivery systems is of great importance for the design of further improved carriers. The first step in the process of intracellular gene delivery is the cellular uptake of the nonviral gene delivery system. Several cellular uptake mechanisms are known. Therefore, the aim of this study was to determine which pathways are involved in uptake and intracellular routing of pDMAEMA- and PEI-based polyplexes in COS-7 cells. Additionally, we would like to know which endocytic pathways contributed most to effective gene delivery. COS-7 cells were used in this study as they are a well-established model cell line for gene transfer research. Both pDMAEMA and PEI have been shown to be able to condense DNA into positively charged polyplexes that bind and transfect cells (15-17). A detectable fraction of PEI 
polyplexes was already taken up within $10 \mathrm{~min}$ and resulted in luciferase expression after $30 \mathrm{~min}$ of incubation, whereas pDMAEMA-based polyplexes acted at a slower pace and showed detectable levels of intracellular fluorescence only after $30 \mathrm{~min}$, and luciferase expression after a $90 \mathrm{~min}$ period of incubation. Moreover, transfection with PEI-polyplexes resulted in higher levels of luciferase gene expression. One of the reasons for the fast uptake and transfection kinetics of PEI polyplexes as compared to the pDMAEMA polyplexes may be the differences in particle size. The larger PEI particles can sediment faster onto the cells than small particles. Moreover, large particles have a bigger payload, which results in delivery of more DNA at similar cell-surface occupation. This may explain why uptake of the larger PEIbased polyplexes is already detectable at an earlier time point compared to the smaller pDMAEMA particles and results in higher levels of reporter gene expression. However, it cannot be excluded that the observed differences in internalization kinetics and gene expression levels may be caused by the utilization of a different internalization pathway of PEI-based polyplexes compared to pDMAEMA-based polyplexes.

To find out which routes of uptake are used by the PEI- and pDMAEMA-based polyplexes in COS-7 cells, colocalization of internalized polyplexes with either transferrin or cholera toxin was studied using spectral bio-imaging (14). Results are not intended to be discussed quantitatively, but are presented to support the findings acquired by means of flow cytometry. Both types of polyplexes showed colocalization with both transferrin and cholera toxin B. It is generally accepted that transferrin is internalized exclusively by clathrin-coated vesicles. Co-localization of polyplexes with transferrin therefore assures that polyplexes make use of the clathrin-dependent uptake route. However, there is some controversy about the uptake route used by cholera toxin (CT). Although some groups have used cholera toxin as a marker for caveolae, other groups demonstrate that CT is taken up by different uptake routes, including clathrindependent as well as caveolae- and clathrin-independent routes (11, 30-32). Partial colocalization of polyplexes with $\mathrm{CT}$, as was seen in this study, makes interpretation of the results therefore difficult. Nevertheless, as not all PEI- or pDMAEMA-based polyplexes co-localized with transferrin, it is very likely that besides clathrin-dependent uptake routes polyplexes make use of other endocytic routes.

To study the route of uptake of the polymer/DNA complexes in a more quantitative way, COS-7 cells were incubated with a panel of inhibitors of different endocytic pathways and analyzed by flow cytometry. Inhibition of macropinocytosis did not affect the total level of uptake of either pDMAEMA- or PEI-based polyplexes. Internalization of both pDMAEMA- and PEI-based polyplexes decreased after incubation with methyl $\beta$-cyclodextrin, which causes cholesterol depletion and thereby affects both the clathrinand caveolae-mediated pathways $(37,38)$. To discriminate between these two pathways more specific inhibitors of clathrin-mediated (chlorpromazine) and of caveolae/lipid raft-mediated (genistein) routing were used. Inhibition of caveolar uptake significantly reduced uptake of PEI polyplexes, but not of pDMAEMA polyplexes. Surprisingly, incubation with chlorpromazine did not affect the internalization of either pDMAEMA- or PEI-based polyplexes. As clear colocalization of both PEI- and pDMAEMA-based polyplexes with transferrin was observed in COS-7 cells in the absence of inhibitors, the lack of reduction in polyplex uptake when the clathrin-dependent pathway was blocked may indicate a certain degree of redundancy in uptake routes. Blocking the clathrin-dependent pathway may result in increased uptake by other endocytic routes. Similar redundancy of endocytic pathways have been demonstrated by other groups before $(40,41)$.

The differences in effectiveness of the two types of polyplexes in the various inhibitor experiments may be due to their differences in size. Polyplexes form a heterogeneous population from which smaller particles can be taken up via the clathrin-mediated pathway and larger particles via the caveolae-mediated pathway. Rejman et al. recently showed that latex beads with a size smaller than $200 \mathrm{~nm}$ were taken up via the clathrin-mediated pathway while larger beads were taken up via the caveolae/lipid raft mediated route (42). Furthermore, histidylated polylysine particles with a size ranging from 70 to $200 \mathrm{~nm}$, were shown to be internalized by HepG3 cells via both the clathrin-dependent and -independent pathway (43).

Previously, Bieber et al. reported that PEI polyplexes accumulate in the lysosomal compartment after cellular uptake (19). However, they only observed co-localization between a fraction of the polyplexes and lysosomal markers. A significant part of the polyplexes resided in vesicles that did not co-localize with lysosomal markers. These results indicate the presence of polyplexes in other vesicles that could well represent caveosomes. Akinc et al. tested the proton sponge hypothesis of PEI and concluded that PEI particles avoid going to the lysosomes, because their surrounding $\mathrm{pH}$ averages 6.1 (20). Based on our findings and the study by Huth et al. (22), it is likely that the vesicles in which Akinc et al. observed their PEI complexes represented a combination of both acidified endosomes/lysosomes and neutral $\mathrm{pH}$ caveosomes.

Both uptake and intracellular routing of gene delivery systems are of major importance to achieve transfection. Therefore, the effects of blocking specific uptake routes on gene expression were also determined. The inhibitors themselves did not negatively influence gene expression directly. Therefore, decrease in transgene expression was caused by the effect of the inhibitors on uptake or intracellular routing of the polyplexes. The expression of luciferase was unaffected when inhibiting macropinocytosis. Inhibition of the clathrin-dependent pathway increased transfection efficiency of both types of polyplexes and therefore clathrin-dependent uptake of polyplexes did not contribute to transfection. Surprisingly, whilst uptake of pDMAEMA was hardly affected by inhibitors of caveolae-mediated routing, gene expression of luciferase was decreased almost completely. This suggests that genistein is not inhibiting cellular uptake, but cellular processing via caveolae, resulting in a reduction of gene expression.

Some controversy about cellular uptake and gene delivery of cationic polymers remains. Kichler et al. showed that incubation of cells with bafilomycin $\mathrm{A} 1$, a specific vacuolar proton pump inhibitor, decreased PEI-mediated gene transfer (44). By inhibiting this vacuolar pump bafilomycin A1 prevents acidification of the internal space of 
several organelles (45). Kichler et al. concluded from their results that acidification of endosomes was important for PEI-mediated transfection. However, they did not demonstrate that their PEI polyplexes were actually taken up via clathrin-mediated endocytosis. Moreover, bafilomycin also acts on a $\mathrm{V}$-type proton pump responsible for acidification of plasmalemmal vesicles, which are created each time a caveolae buds off from the cell surface and was even found associated with caveolae (46). Therefore effects of bafilomycin on caveolar uptake and trafficking of PEI polyplexes cannot be excluded from their work.

Gersdorff et al. recently showed that chlorpromazineinduced inhibition of the clathrin-dependent uptake route resulted in loss of transfection with PEI polyplexes in several different cell lines, suggesting that the clathrin-dependent uptake route is important for successful transfection (48). Careful evaluation of the results, however, revealed that most of these experiments were performed at inhibitor concentrations that also showed direct toxicity to cells, which may have partially accounted for the loss of transfection.

On the other hand, in line with our observations Kopatz et al., show that PEI polyplexes are taken up by HeLa cells after initial binding to heparin sulfate proteoglycans, followed by uptake into cholesterol-rich rafts (47). These results correspond to those obtained by Huth et al. (22). Although in this study the importance of caveolar uptake on effective gene delivery with polyplexes was only studied in COS-7 cells, similar findings were recently reported for other cell types, indicating that this is a more general phenomenon (23).

Taken together, our data demonstrate that uptake of pDMAEMA and PEI polyplexes in COS-7 cells is mediated via different uptake routes, including both the clathrin- and caveolae-mediated pathway. However, only the DNA imported via caveolar uptake is expressed. These results offer the possibility of developing new strategies for improvements in gene delivery through non-viral means. Until now the focus was mainly on the escape from the acidic environment of endosomes, but with the present data we conclude that more emphasis should be put on intracellular trafficking via the caveolar/lipid raft pathway.

\section{REFERENCES}

1. Y. W. Cho, J. D. Kim, and K. Park. Polycation gene delivery systems: escape from endosomes to cytosol. J. Pharm. Pharmacol. 55:721-734 (2003).

2. D. Lechardeur, A. S. Verkman, and G. L. Lukacs. Intracellular routing of plasmid DNA during non-viral gene transfer. $A d v$. Drug Deliv. Rev. 57:755-767 (2005).

3. M. Belting, S. Sandgren, and A. Wittrup. Nuclear delivery of macromolecules: barriers and carriers. Adv. Drug Deliv. Rev. 57:505-527 (2005).

4. I. A. Khalil, K. Kogure, H. Akita, and H. Harashima. Uptake pathways and subsequent intracellular trafficking in nonviral gene delivery. Pharmacol. Rev. 58:32-45 (2006).

5. K. Takeiand and V. Haucke. Clathrin-mediated endocytosis: membrane factors pull the trigger. Trends Cell Biol. 11:385-391 (2001).

6. R. G. Anderson. The caveolae membrane system. Annu. Rev. Biochem. 67:199-225 (1998).

7. P. U. Le and I. R. Nabi. Distinct caveolae-mediated endocytic pathways target the Golgi apparatus and the endoplasmic reticulum. J. Cell Sci. 116:1059-1071 (2003).
8. I. R. Nabi and P. U. Le. Caveolae/raft-dependent endocytosis. $J$. Cell Biol. 161:673-677 (2003).

9. L. Pelkmans, J. Kartenbeck, and A. Helenius. Caveolar endocytosis of simian virus 40 reveals a new two-step vesiculartransport pathway to the ER. Nat. Cell Biol. 3:473-483 (2001).

10. M. J. Duncan, J. S. Shin, and S. N. Abraham. Microbial entry through caveolae: variations on a theme. Cell. Microbiol. 4:783791 (2002).

11. W. I. Lencer, T. R. Hirst, and R. K. Holmes. Membrane traffic and the cellular uptake of cholera toxin. Biochim. Biophys. Acta 1450:177-190 (1999).

12. L. Pelkmans and A. Helenius. Endocytosis via caveolae. Traffic 3:311-320 (2002).

13. J. A. Swanson and C. Watts. Macropinocytosis. Trends Cell Biol. 5:424-428 (1995).

14. U. Huth, A. Wieschollek, Y. Garini, R. Schubert, and R. Peschka-Suss. Fourier transformed spectral bio-imaging for studying the intracellular fate of liposomes. Cytometry $A$ 57: 10-21 (2004).

15. O. Boussif, F. Lezoualc'h, M. A. Zanta, M. D. Mergny, D. Scherman, B. Demeneix, and J. P. Behr. A versatile vector for gene and oligonucleotide transfer into cells in culture and in vivo: polyethylenimine. Proc. Natl. Acad. Sci. USA. 92 7297-7301 (1995).

16. P. van de Wetering, J. Y. Cherng, H. Talsma, and W. E. Hennink. Relation between transfection efficiency and cytotoxicity of poly(2-dimethylamino)ethyl methacrylate/plasmid complexes. J. Control. Release 49:59-69 (1997).

17. N. J. Zuidam, G. Posthuma, E. T. de Vries, D. J. Crommelin, W. E. Hennink, and G. Storm. Effects of physicochemical characteristics of poly(2-(dimethylamino)ethyl methacrylate)-based polyplexes on cellular association and internalization. J. Drug Target. 8:51-66 (2000).

18. W. T. Godbey, K. K. Wu, and A. G. Mikos. Tracking the intracellular path of poly(ethylenimine)/DNA complexes for gene delivery. Proc. Natl. Acad. Sci. U. S. A. 96:5177-5181 (1999).

19. T. Bieber, W. Meissner, S. Kostin, A. Niemann, and H. Elsasser Intracellular route and transcriptional competence of polyethylenimine-DNA complexes. J. Control. Release 82:441 (2002).

20. A. Akinc, M. Thomas, A. M. Klibanov, and R. Langer. Exploring polyethylenimine-mediated DNA transfection and the proton sponge hypothesis. J. Gene Med. 7:657-663 (2004).

21. N. D. Sonawane, F. C. Szoka, Jr., and A. S. Verkman. Chloride accumulation and swelling in endosomes enhances DNA transfer by polyamine-DNA polyplexes. J. Biol. Chem. 278:4482644831 (2003).

22. S. Huth, J. Lausier, S. W. Gersting, C. Rudolph, C. Plank, U. Welsch, and J. Rosenecker. Insights into the mechanism of magnetofection using PEI-based magnetofectins for gene transfer. J. Gene Med. 6:923-936 (2004).

23. J. Rejman, A. Bragonzi, and M. Conese. Role of clathrin- and caveolae-mediated endocytosis in gene transfer mediated by lipo- and polyplexes. Mol. Ther. 12:468-474 (2005).

24. S. Grosse, Y. Aron, G. Thevenot, D. Francois, M. Monsigny, and I. Fajac. Potocytosis and cellular exit of complexes as cellular pathways for gene delivery by polycations. J. Gene. Med. (2005).

25. J. Y. Cherng, P. van de Wetering, H. Talsma, D. J. Crommelin, and W. E. Hennink. Effect of size and serum proteins on transfection efficiency of poly ((2-dimethylamino)ethyl methacrylate)-plasmid nanoparticles. Pharm. Res. 13:1038-1042 (1996).

26. M. A. van der Aa, G. A. Koning, C. d'Oliveira, R. S. Oosting, K. J. Wilschut, W. E. Hennink, and D. J. Crommelin. An NLS peptide covalently linked to linear DNA does not enhance transfection efficiency of cationic polymer based gene delivery systems. J. Gene Med. 7:208-217 (2004).

27. C. Arigita, N. J. Zuidam, D. J. Crommelin, and W. E. Hennink. Association and dissociation characteristics of polymer/DNA complexes used for gene delivery. Pharm. Res. 16:1534-1541 (1999).

28. S. Rothenberger, B. J. Iacopetta, and L. C. Kuhn. Endocytosis of the transferrin receptor requires the cytoplasmic domain but not its phosphorylation site. Cell 49:423-431 (1987).

29. I. Killisch, P. Steinlein, K. Romisch, R. Hollinshead, H. Beug, and G. Griffiths. Characterization of early and late endocytic 
compartments of the transferrin cycle. Transferrin receptor antibody blocks erythroid differentiation by trapping the receptor in the early endosome. J. Cell Sci. 103:211-232 (1992).

30. K. Sandvig, and B. van Deurs. Membrane traffic exploited by protein toxins. Annu. Rev. Cell Dev. Biol. 18:1-24 (2002).

31. O. O. Glebov and B. J. Nichols. Lipid raft proteins have a random distribution during localized activation of the T-cell receptor. Nat. Cell Biol. 6:238-243 (2004).

32. M. Kirkham, A. Fujita, R. Chadda, S. J. Nixon, T. V. Kurzchalia, D. K. Sharma, R. E. Pagano, J. F. Hancock, S. Mayor, and R. G. Parton. Ultrastructural identification of uncoated caveolinindependent early endocytic vehicles. J. Cell Biol. 168:465-476 (2005).

33. U. Huth, R. Schubert, and R. Peschka-Suss. Investigating the uptake and intracellular fate of $\mathrm{pH}$-sensitive liposomes by flow cytometry and spectral bio-imaging. J. Control. Release 110(3): 490-504 (2006).

34. N. Araki, M. T. Johnson, and J. A. Swanson. A role for phosphoinositide 3-kinase in the completion of macropinocytosis and phagocytosis by macrophages. J. Cell Biol. 135:1249-1260 (1996).

35. H. Shpetner, M. Joly, D. Hartley, and S. Corvera. Potential sites of PI-3 kinase function in the endocytic pathway revealed by the PI-3 kinase inhibitor, wortmannin. J. Cell Biol. 132:595-605 (1996).

36. E. P. Kilsdonk, P. G. Yancey, G. W. Stoudt, F. W. Bangerter, W. J. Johnson, M. C. Phillips, and G. H. Rothblat. Cellular cholesterol efflux mediated by cyclodextrins. J. Biol. Chem. 270:17250-17256 (1995).

37. S. K. Rodal, G. Skretting, O. Garred, F. Vilhardt, B. van Deurs, and K. Sandvig. Extraction of cholesterol with methyl-beta-cyclodextrin perturbs formation of clathrin-coated endocytic vesicles. Mol. Biol. Cell 10:961-974 (1999).

38. A. Subtil, I. Gaidarov, K. Kobylarz, M. A. Lampson, J. H. Keen, and T. E. McGraw. Acute cholesterol depletion inhibits clathrincoated pit budding. Proc. Natl. Acad. Sci. U. S. A. 96:6775-6780 (1999).
39. R. G. Parton, B. Joggerst, and K. Simons. Regulated internalization of caveolae. J. Cell Biol. 127:1199-1215 (1994).

40. H. Damke, T. Baba, A. M. van der Bliek, and S. L. Schmid. Clathrin-independent pinocytosis is induced in cells overexpressing a temperature-sensitive mutant of dynamin. J. Cell Biol. 131:69-80 (1995).

41. B. J. Nichols. A distinct class of endosome mediates clathrinindependent endocytosis to the Golgi complex. Nat. Cell Biol. 4:374-378 (2002).

42. J. Rejman, V. Oberle, I. S. Zuhorn, and D. Hoekstra. Sizedependent internalization of particles via the pathways of clathrin- and caveolae-mediated endocytosis. Biochem. J. 377:159-169 (2004).

43. C. Goncalves, E. Mennesson, R. Fuchs, J. P. Gorvel, P. Midoux, and C. Pichon. Macropinocytosis of polyplexes and recycling of plasmid via the clathrin-dependent pathway impair the transfection efficiency of human hepatocarcinoma cells. Mol. Ther. 10:373-385 (2004).

44. A. Kichler, C. Leborgne, E. Coeytaux, and O. Danos. Polyethylenimine-mediated gene delivery: a mechanistic study. $J$. Gene Med. 3:135-144 (2001).

45. E. J. Bowman, A. Siebers, and K. Altendorf. Bafilomycins: a class of inhibitors of membrane ATPases from microorganisms, animal cells, and plant cells. Proc. Natl. Acad. Sci. USA. 85: 7972-7976 (1988).

46. C. Mineo and R. G. Anderson. A vacuolar-type proton ATPase mediates acidification of plasmalemmal vesicles during potocytosis. Exp. Cell Res. 224:237-242 (1996).

47. I. Kopatz, J. S. Remy, and J. P. Behr. A model for non-viral gene delivery: through syndecan adhesion molecules and powered by actin. J. Gene Med. 6:769-776 (2004).

48. K. von Gersdorff, N. N. Sanders, R. Vandenbroucke, S. C. De Smedt, E. Wagner, and M. Ogris. The internalization route resulting in successful gene expression depends on both cell line and polyethylenimine polyplex type. Mol. Ther. 14:745-753 (2006). 